Dita B. Vizoso · Dieter Ebert

\title{
Mixed inoculations of a microsporidian parasite with horizontal and vertical infections
}

\begin{abstract}
Mixed infections, where more than one parasite genotype is present in a single host, have been suggested to be an important factor in host-parasite interactions. As the host represents a limited resource, co-infecting parasite genotypes are expected to be under resource competition. Competition will not only modify the survival of the co-infecting genotypes, but is also likely to affect total within-host parasite growth as well as host survival and reproduction. We measured parasite infectivity and spore production of seven isolates of Octosporea bayeri (Microsporidia) and their effect on the reproduction and longevity of its host Daphnia magna (Cladocera), after single- or double-isolate inoculations through vertical and horizontal transmission. Doubleisolate inoculations increased parasite infectivity and total spore production in horizontal infections, but had no significant effect on host reproduction or longevity. The increase in spore production in double-isolate inoculations was not found when infections occurred vertically. Our results suggest that, depending on the way the infection was acquired, within-host reproduction can increase as a result of parasite genetic diversity, without necessarily affecting the host's fitness. Whether this challenges the current views of virulence evolution depends on the definitions used.
\end{abstract}

Keywords Multiple infections · Parasite fitness · Host fitness $\cdot$ Pathogenicity $\cdot$ Dose effect .

Octosporea bayeri $\cdot$ Daphnia magna

D. B. Vizoso · D. Ebert

Département de Biologie, Unité d'Ecologie and Evolution,

Université de Fribourg, Chemin du Musée 10,

1700 Fribourg, Switzerland

Present address: D. B. Vizoso ( $\square)$

Institute of Zoology and Limnology,

University of Innsbruck, Technikerstrasse 25,

6020 Innsbruck, Austria

E-mail: dita.vizoso@uibk.ac.at

Fax: + 43-512-5072930

\section{Introduction}

In many infectious diseases, hosts are naturally infected with more than one genotype of the same parasite. This within-host parasite genetic diversity may alter the immediate outcome of host-parasite interactions, as suggested by models (e.g. Antia et al. 1996; Hellriegel 1992) and experiments (e.g. Davies et al. 2002; Wedekind and Ruetschi 2000; Wille et al. 2002). On a longer time-scale, host and parasite evolution is also predicted to be affected by mixed-genotype infections (e.g. Bremermann and Pickering 1983; Frank 1992). One important aspect of mixed infections is its potential for within-host competition (Read and Taylor 2001). Co-infecting parasites are likely to compete for the same resource, regardless of whether they are genetically identical or not. If they are identical, density dependence may have a negative effect on development, growth, reproduction, and survival of the parasite and may also influence host well being (Ebert et al. 2000). In genetically diverse infections, genotypes with different strategies for resource exploitation might be under competition. When more than one parasite genotype infects a host, genotypes with a prudent exploitation strategy (which might be otherwise favoured in singlegenotype infections) may be outcompeted by genotypes that exploit the host in a faster and less prudent way (Frank 1996). Although an increase in the damage inflicted to the host might then decrease the relative benefits of a particular infection for all the co-infecting parasites (e.g. by reducing the host's longevity), the prudent genotypes would do disproportionately worse and may thus be eliminated as the result of competition (Bremermann and Pickering 1983; Read and Taylor 2001). Following this rationale, different theoretical approaches concluded that despite the between-host selective pressure to use the host prudently, multiple infections will select for higher levels of within-host reproduction (Bonhoeffer and Nowak 1994; Frank 1996; May and Nowak 1995; van Baalen and Sabelis 1995). 
Herre (1993, 1995), showed that the potential for multiple infections increases parasite virulence (in this case defined as the reduction in the lifetime reproductive success of the host owing to the parasite), thought to be a consequence of increased within-host reproduction. Under certain conditions, however, more prudent parasite genotypes may overgrow and even outcompete more rapacious ones, resulting in a reduced disease severity in the presence of multiple infections (Read and Taylor 2001). It also has been shown that viruses can evolve strategies to increase their fitness without a concomitant increase in the damage to the host, in a sort of hyper-parasitism where one product of virus metabolism is consumed by certain virus genotypes which then cease to produce it themselves (Turner and Chao 1999). In this latter example, it has been suggested that the behaviour of co-infecting parasites can be characterized by a "collective action" perspective, where the existence of a "common good" could even lead to cooperation between genotypes, reducing host exploitation and, consequently, the harm to the host (Brown et al. 2002). Finally, when including sub-lethal effects of parasitism such as reduced host growth, models of multiple infections can also predict a decrease in the within-host parasite growth rate (Schjørring and Koella 2003).

Whereas we have avoided using the term "virulence", most of the studies on multiple infections (including the aforementioned) talk about the effects of parasite genetic diversity on virulence, with the resulting debate on whether multiple infections will increase it or not. The definition of the term virulence, however, is "used in a diffuse manner" (Knolle 1989), and varies between and within empirical and theoretical studies. It is beyond the scope of this paper to either review the definitions of virulence or suggest a general definition (see Read 1994; Poulin and Combes 1999 for two perspectives), but it is necessary to point out these discrepancies, as they render difficult the interpretation of empirical data in the light of the current theories (see e.g. Day 2002a).

From an evolutionary perspective, "virulence" usually stands for the parasite-induced reduction in host fitness (Herre 1993), and therefore is neither an attribute of the parasite nor of the host alone but the result of an interaction between the two (Ebert 1994; Herre 1995; Read 1994). To avoid confusion we will refer to the detrimental effects of the parasite on the host as pathogenicity. As different life history traits of the host and the parasite are evolutionarily important (e.g. Perlman and Jaenike 2003) we assess the effects of multiple infections on these components separately, namely parasite infectivity, parasite within-host growth, host longevity and host reproduction.

Within-host interactions between parasite genotypes have been shown to affect parasite infectivity and load (e.g. Wedekind and Ruetschi 2000; Wille et al. 2002), warning us that the outcome might depend on the particular parasite genotypes co-infecting the host. Most studies on mixed infections have, however, used only two parasite genotypes, and compared the outcome of the two single-genotype infections with the double-genotype infections (but see Wedekind and Ruetschi 2000; Wille et al. 2002). Thus, it is not possible to distinguish between the effects of double infections per se and the effect of a specific combination of two genotypes. Another important aspect to consider is the total amount of parasites present in the inoculation. Dose effects may not be the same for different parasite genotypes, and the results of mixed inoculations might simply represent changes in the size of the inoculum (e.g. Taylor et al. 1997).

A further complication arises when considering different infection routes. Many parasites transmit to new hosts not only horizontally (as is assumed in most of the theory modelling multiple infections, see van Baalen 2000 for an exception) but also vertically, from parent to offspring. When parasite transmission occurs exclusively vertically, the fitness of a parasite becomes linked to that of its host, with natural selection favouring a decrease in pathogenicity (Bull 1994; Bull et al. 1991; Frank 1996). In those cases where vertical transmission is internal (e.g. transovarial transmission of microsporidia), it might also affect the outcome of within-host competition. This is because only a limited number of parasites will have access to the new host, increasing the advantage of parasite genotypes that gain access to the eggs or embryo (without a necessary increase in pathogenicity), and reducing the heterogeneity of the subsequent infection.

The microsporidium Octosporea bayeri Jírovec (1936) is a pathogen of the planktonic cladoceran Daphnia magna Strauss that is capable of both horizontal and vertical infection (Vizoso et al., in press). O. bayeri has a high prevalence in D. magna rock-pool populations, which are part of a large metapopulation in southern Finland (Ebert et al. 2001; Green 1957), with a high degree of spatial heterogeneity and migration (Ebert et al. 2002). Parasite isolates from different populations vary in their within-host reproduction and pathogenicity (D. B. Vizoso, unpublished results). The dynamics of the system suggest that the presence of different parasite genotypes in a single D. magna population might be frequent, and thus this is a suitable system in which to test for the effect of mixed inoculations and its interaction with the route of infection. Here, we investigate the effects of mixed-genotype inoculations on parasite and host life history using all possible pairwise combinations of seven isolates of $O$. bayeri to infect a single clone of D. magna. The hosts were infected horizontally or vertically to test for possible interactions between transmission route and parasite diversity. We also assessed the effect of inoculum size (dose effect) on the studied variables.

\section{Materials and methods}

\section{Study organisms}

We used seven isolates of the microsporidium $O$. bayeri (Ob10 - Ob16), each from a different natural rock-pool 
D. magna population of different islands in the Tvärminne Archipelago in southern Finland. To control for the effects of host genetic diversity, we used a single clone of D. magna, which was outbred from uninfected individuals from rock-pool populations other than those of the parasites, but from the same area. We thus expect that although the parasite isolates may not have been exposed to this particular clone, it will be as similar as possible to the clones they were collected in. Individuals of the same clone of D. magna are genetically identical, and thus we can study differences in the parasite while keeping the host background constant. Laboratory lines of each parasite isolate in this host clone were then created by exposing 5-day-old female Daphnia (all from the same host clone, i.e. lacking genetic variation) to parasite spores. Each Daphnia was kept individually in $2.5 \mathrm{ml}$ of artificial medium (Klüttgen et al. 1994; modified after Ebert et al. 1998) with a spore solution of 40,000 spores $/ \mathrm{ml}$ for 5 days, and then transferred to $100 \mathrm{ml}$ medium until the second clutch was produced. The presence of spores in the exposed Daphnia was then assessed under the microscope, and the offspring of the infected individuals distributed among four replicate 400-ml jars. To control for possible maternal effects, these isolate lines were kept for at least five generations under identical, standard laboratory conditions: in artificial medium, fed regularly with the unicellular green algae Scenedesmus sp., in a light:dark cycle of $8: 16 \mathrm{~h}$, and at $20^{\circ} \mathrm{C}$. The lack of molecular markers prevents a direct assessment of the genetic differences of the parasite isolates. However, differences in the effects of infection (parasite spore load, host longevity and reproduction) between the parasite isolates have been found while controlling for host genetic diversity (using the same host clone, see above) and maternal or environmental effects (unpublished results), strongly suggesting the existence of genetic diversity between the parasite isolates used.

\section{Experiment}

To assess the effects of increasing the diversity of inoculations on parasite and host life histories we performed a life-table experiment. In order to simultaneously survey vertically and horizontally infected individuals, we had to perform two subsequent inoculations: one for the mothers of the vertically infected Daphnia and a second one for the horizontally infected Daphnia. In both cases, all hosts stem from a few, genetically identical mothers (from the host clone, see above) and were randomised among all the treatments.

\section{First inoculation}

We homogenised dying individuals to prepare spore solutions from all seven parasite isolates. We then inoculated 3-day-old uninfected Daphnia (all from the experimental host clone, i.e. with negligible genetic variation) either with single isolates (SI) or with one of the 21 pairwise isolate combinations (double isolate; DI). DI inoculations were produced by adding 50,000 spores of each isolate in succession. In SI inoculations we added the same isolate twice. Thus, for isolates A and $\mathrm{B}$, half the DI inoculation (AB) was A and the other half was $\mathrm{B}$, while the SI inoculations can be considered as twice the same isolate, AA and BB. The infection procedure was as described above. The surviving animals were placed in groups of three in 100-ml jars, and the second clutch was used for the vertical treatments of the experiment. The SI-infected mothers were then homogenised to prepare seven spore solutions to inoculate the females in the horizontal treatments of the experiment, and thus served also as spore donors. A similar number of uninfected animals underwent the same procedures (with a placebo made of uninfected dead Daphnia instead of spore solutions) to produce the uninfected Daphnia inoculated in the horizontal treatments.

\section{Second inoculation}

Horizontal SI treatments were produced by exposing animals from the uninfected lines to two successive doses of the same isolate (seven treatments), whereas horizontal DI treatments received successive doses of two different isolates (all pairwise combinations of the seven isolates, 21 treatments). The 28 vertical treatments (i.e. the offspring of the animals infected in the first inoculation, seven SI and $21 \mathrm{DI}$ ), and an uninfected control received a placebo of uninfected Daphnia. For each treatment we used ten D. magna, comprising a total of 560 animals in the SI-DI comparison, plus 20 animals as uninfected controls. Treatments with half the spore dose of SI inoculations (five isolates, 15 animals per treatment for a total of 75) were added to distinguish between the effect of increasing parasite genetic diversity and the effect of increasing parasite dose. All inoculations were done in 24-well cell-culture plates with four treatments per plate, randomly distributed. Animals were transferred to $100 \mathrm{ml}$ medium after 5 days. Thereafter, they were transferred to fresh medium every third day and fed with $2 \times 10^{6}$ algae cells. This food regime is low in comparison with other experiments using D. magna, and was chosen to enhance any possible effects of resource competition between parasite genotypes.

\section{Life table}

The animals were monitored daily, and age at maturity, number of offspring produced, and longevity (time until death) were recorded. Dead animals were frozen in $0.5 \mathrm{ml}$ medium at $-20^{\circ} \mathrm{C}$ for later spore counts. To determine the spore load (number of spores per host) the Daphnia were thawed, homogenised with a plastic pestle, and resuspended. The concentration of spores in these suspensions was determined with a cell counting chamber (haemocytometer) using a phase-contrast 
microscope. The three different spore types described for $O$. bayeri (Vizoso and Ebert 2004; Vizoso et al., in press) were scored, and spore load was calculated as the sum of the three spore types.

\section{Inoculate diversity}

The lack of molecular markers for $O$. bayeri prevents us from directly assessing the genetic diversity of each parasite isolate and the existence of genetic differences between the different isolates. On one hand, as each parasite isolate was obtained from a field-collected, infected host, it may contain several parasite genotypes (therefore the use of "isolate" instead of "strain" or "clone"). On the other hand, although it is unlikely that parasites from pools in different islands are genetically identical, we cannot exclude this possibility. However, previous studies have shown that parasite isolates of $O$. bayeri from the same area behave as discrete entities, and differ in several aspects of the infection (Vizoso 2003; Vizoso and Ebert 2004; Vizoso and Ebert, in review) suggesting that the genetic diversity between isolates is considerably larger than that within isolates. By controlling host genetic diversity and environmental (maternal) effects, we consider that any differences between parasite isolates in the present study would reflect the existence of such genetic differences. In a conservative way, the double-isolate inoculations simply increase the chances of increasing the genetic diversity of the infection.

\section{Data analysis}

To test whether the infectivity, spore production, and the effects on the host of the parasite isolates varied if they were inoculated singly or in pairs, we compared SI and DI inoculates for each isolate. We used two-tailed paired $t$-tests on the mean responses of isolates when infected singly versus their mean responses when infected in pairs, within each infection route ( $n=7$ isolates). To maintain the independence of the data points (the means of treatments within the same isolate) we randomly assigned half of the hosts of each combination $\mathrm{AB}$ as the DI group to compare with $\mathrm{AA}$, and the other half as the DI group to compare with BB.

We tested a posteriori whether the infectivity of DI inoculations was higher than that of the isolate with the highest infectivity when inoculated singly. We used a one-tailed paired $t$-test on the infectivity of isolates when infected singly versus the average infectivity of the pairs in which the other component was less infective. In other words, AA was paired with all DI groups Ax, given that the infectivity of AA was higher than the infectivity of $x x$. We used the same random assignment of hosts from the DI groups described in the previous paragraph.

As we are interested in testing the effect of mixed inoculations, the presence of dose-dependent effects needs to be considered. To determine whether there is an effect of spore dose on the performance of the isolates, we infected an additional treatment group with half the spore dose, using five of the single isolates (restricted to horizontal infections). We then compared the horizontally infected SI treatments (full versus half dose) using a two-way ANOVA. We further tested the effect of spore dose on infection frequency using a two-way logistic regression with dose and parasite isolate as factors.

Data were transformed whenever the assumptions of the tests performed were not met. Parasite isolate was treated as random factor when appropriate. All analyses were done using JMP 4 (SAS Institute 2000).

\section{Results}

\section{Spore dose}

The proportion of hosts that became infected after SI horizontal inoculations did not differ among the two dose levels (full vs. half spore dose; Fig. 1a, logistic regression analysis, Wald $\chi^{2}$ after removing interaction $=0.39, P=0.53$ ), parasite isolate (Wald $\chi^{2}$ after removing interaction $=1.27, P=0.87$ ), or their interaction (Wald $\chi^{2}=0.17, P=0.99$ ). Likewise, spore dose had no significant effect on parasite spore load (Fig. 1b, Table 1), which varied strongly between parasite isolates. Neither host reproduction (Fig. 1c) nor host longevity (Fig. 1d) were significantly affected by spore dose, parasite isolate, or their interaction (Table 1).

\section{Mixed versus pure inoculations}

DI inoculations had a significantly higher infectivity than SI inoculations, when tested overall (Table 2, Fig. 2a). Spore production, measured as the total spore load at host death, also increased with parasite heterogeneity (Fig. 2b), the difference being substantial and significant in horizontal infections (an average of 18.7\%, Table 2), but absent when the infection occurred vertically. Despite the observed increase in spore production, hosts from DI inoculations did not have a lower host reproduction or longevity, regardless of the infection route (Fig. 2c, d, Table 2). The mean spore production of isolates correlated positively with host reproduction when inoculated horizontally, both in SI and DI inoculations (Fig. 3a, Spearman's rho for SI $=0.82, P=0.023, n=7$; for $\mathrm{DI}=0.75, P=0.052, n=7$ ), while a trend in the opposite direction was found in vertical infections (Fig. 3b, Spearman's rho for $\mathrm{SI}=-0.43, P=0.33, n=7$; for $\mathrm{DI}=-0.61, P=0.15, \mathrm{n}=7)$. As host genetic diversity and maternal effects are controlled in our experimental design, the most likely explanation for the differences between the SI inoculations shown in Fig. 2 is that the parasite isolates used actually differ genetically.

In order to determine whether the observed increase in infectivity and spore load in DI inoculations was due 

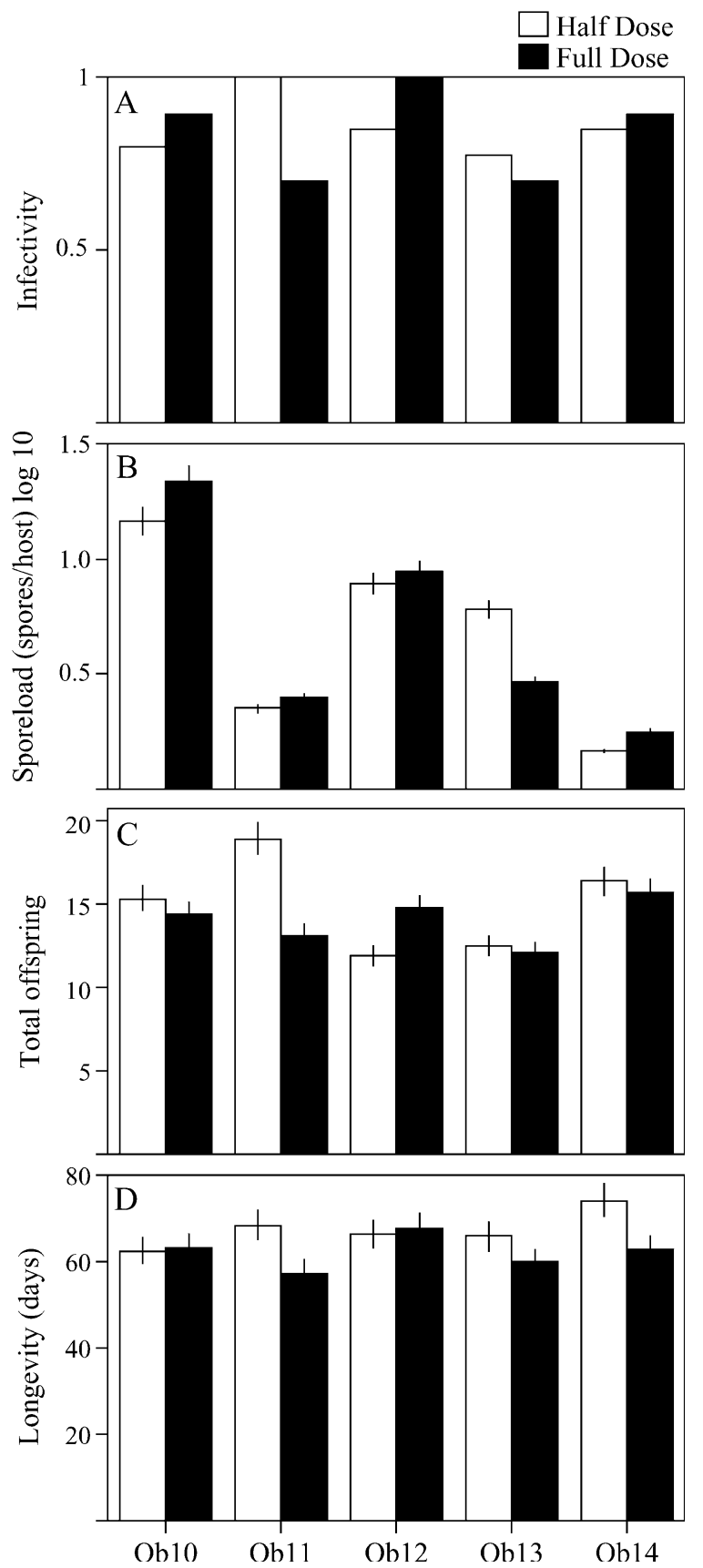

Fig. 1a-d Effect of spore-dose on parasite and host life history for five different isolates of Octosporea bayeri (Ob10-14) infecting Daphnia magna. a Infectivity, as the proportion of infected hosts; b spore load (no. of spores/host) at host death. The data shown are the back-transformed least-squares means from the two-way ANOVA (see Table 1); c lifetime host reproduction (number of clonal offspring); d host longevity (days until death). c, d Leastsquares means from two-way ANOVAs with spore-dose and parasite isolate (Table 1) for isolates Ob10-14, inoculated with a half dose (white columns) or full dose (black columns). Bars indicate SEs

to the isolate of the pair with higher infectivity or spore load, we repeated the paired $t$-test approach, but using the isolate of that pair with the highest infectivity or spore load (see Materials and methods). Both the overall
Table 1 Two-way ANOVAs of the effects of spore dose and parasite isolate on host and parasite life history traits. Significant effects in italics

\begin{tabular}{lllll}
\hline Source & $N$ & MS & $F$-ratio & $P>\mathrm{F}$ \\
\hline Total spore load & & & & \\
$\quad$ Dose & 2 & 0.008 & 0.08 & 0.78 \\
$\quad$ Isolate & 5 & 1.87 & 19.5 & 0.007 \\
$\begin{array}{l}\text { Dosexisolate } \\
\quad \text { Error }\end{array}$ & 97 & 0.09 & 0.56 & 0.69 \\
$\quad$ Host lifetime reproduction & 0.17 & & \\
$\quad$ Dose & 2 & $21.9^{\mathrm{b}}$ & 0.67 & 0.42 \\
$\quad$ Isolate & 5 & $63.3^{\mathrm{b}}$ & 1.93 & 0.11 \\
$\quad$ Dosexisolate & & 47.1 & 1.47 & 0.22 \\
$\quad$ Error & 101 & $32.7^{\mathrm{b}}$ & & \\
$\quad$ Host longevity & & & & \\
$\quad$ Dose & 2 & $696.6^{\mathrm{b}}$ & 1.47 & 0.23 \\
$\quad$ Isolate & 5 & $120.0^{\mathrm{b}}$ & 0.25 & 0.91 \\
Dosexisolate & & 500.1 & 1.06 & 0.38 \\
$\quad$ Error & 114 & $474.5^{\mathrm{b}}$ & & \\
\end{tabular}

${ }^{\mathrm{a}}$ Tested over the $\log _{10}$-transformed data

${ }^{\mathrm{b}}$ Error SS after pooling the interaction SS (after Sokal and Rohlf 1998, p 337)

Table 2 Paired $t$-tests of the differences between double isolate (DI) and single isolate (SI) means for each parasite isolate. The percentage of difference relative to the observed values, its direction and the test results are shown for horizontal $(h)$ and vertical $(v)$ treatments. $d f=6(7$ pure isolates -1$)$ in all tests. Significant effects in italics

\begin{tabular}{lccll}
\hline & & $\% \Delta$ & $|t|$-ratio & $P>|t|$ \\
\hline Infectivity & $\mathrm{h}$ & +17.6 & 2.73 & 0.034 \\
Total spore load & $\mathrm{h}$ & +18.7 & 2.51 & 0.046 \\
& $\mathrm{~V}$ & +1.33 & 0.40 & 0.71 \\
Host lifetime reproduction & $\mathrm{h}$ & +9.53 & 0.61 & 0.56 \\
& $\mathrm{~V}$ & -12.3 & 0.59 & 0.57 \\
Longevity & $\mathrm{h}$ & +9.11 & 1.03 & 0.34 \\
& $\mathrm{v}$ & -3.62 & 0.54 & 0.61 \\
\hline
\end{tabular}

increase in infectivity and in spore load were maintained when restricting the comparison of the DI inoculations to the "best" isolate (Fig. 4, Table 3). Again, no increase in spore load was found for vertical infections (Fig. 4b, Table 3). The parasite isolates Ob15 in horizontal infections and Ob13 in vertical infections, had the lowest infectivity and spore load and thus cannot be included in the analysis, resulting in a lower number of replicates $(n=6)$ than the previous SI-DI comparisons.

\section{Discussion}

Spore dose

Multiple infections go hand in hand with a change in either the total inoculum size or the inoculum size of each isolate of a pair. Therefore, we tested for dose effects to determine to what extent our multiple infection experiments are confounded by them. Halving the spore dose of $O$. bayeri when inoculating D. magna had 
Fig. 2a-d Parasite and host life-histories in infections with the seven $O$. bayeri isolates when infected singly or in pairs. Points represent the means for each isolate when infected with another (double isolate; $D I$, vertical axis) versus the means when infected singly (single isolate; SI, horizontal axis). DI versus SI values shown for horizontal (open circles) and vertical (filled circles) treatments. Dashed lines indicate $\mathrm{DI}=$ SI. a Parasite infectivity (infected/inoculated hosts); b parasite spore load (number of spores/host) at host death; c lifetime host reproduction (number of clonal offspring); d host longevity (days until death)
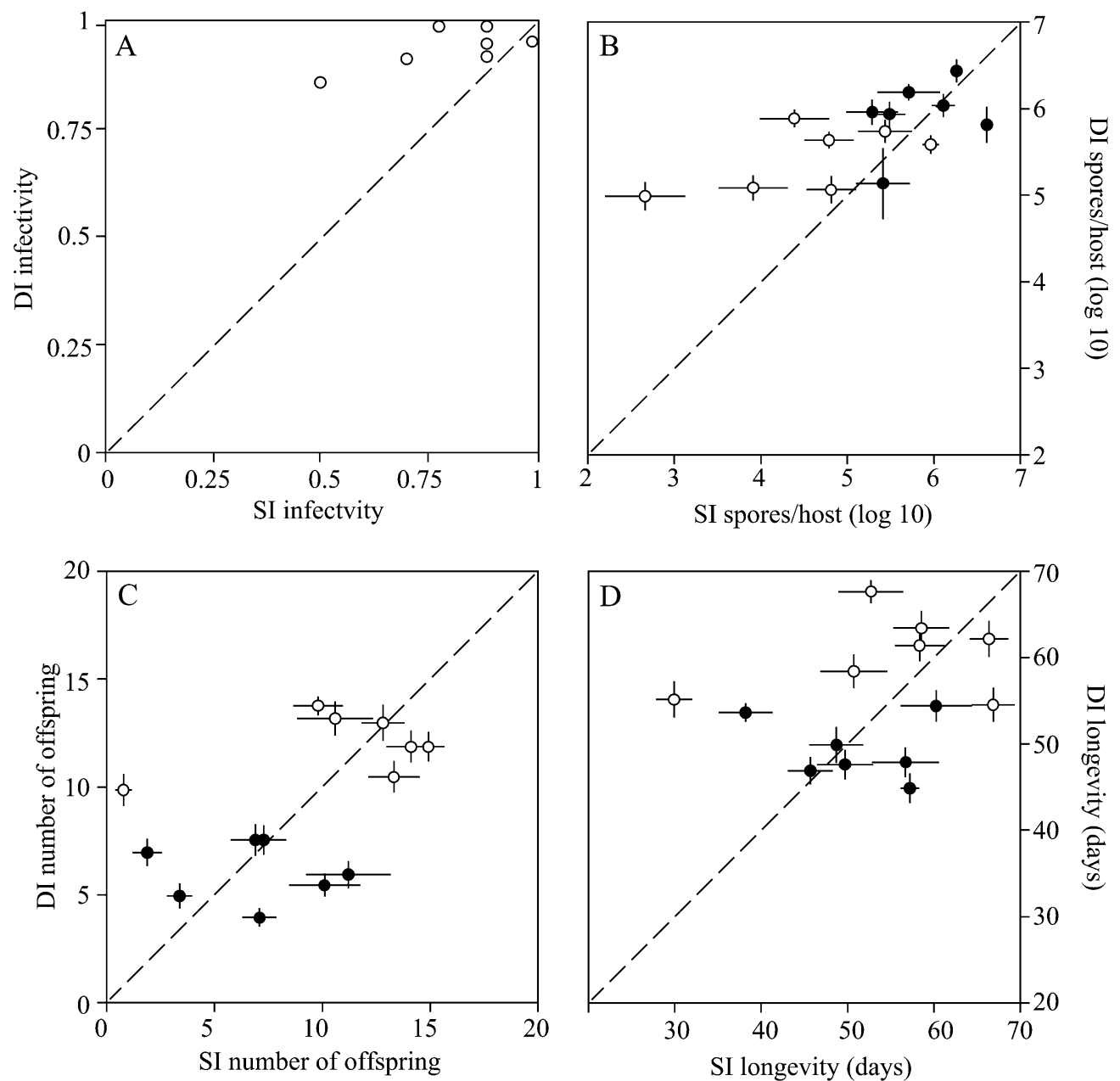

no significant effect on infectivity or spore load. This seems to contrast with other microparasites of D. magna, for which dose effects have been found (Ebert et al. 2000). These other experiments, however, involved much larger differences in the inoculum size than ours. The lack of a dose effect in the present study may thus reflect a small difference in the doses involved, with the effect on host or parasite performance being too small to be detected. Alternatively, a larger number of parasites might indeed have been acquired in the full-dose treatments, but this increment in initial parasite load did not affect the final spore production. Spore load will not necessarily be affected by the number of parasites entering the host (e.g. Ebert et al. 2000). In the case of $O$. bayeri, the spores are accumulated in the host until host death, and thus spore load may be limited by host resources and space. Alternatively, horizontal transmission in $O$. bayeri may obey a saturating function (e.g. Ebert et al. 2000), with no increase in spore uptake beyond a certain threshold dose, with our half-dose being already at, or beyond, that threshold. Thus, in the context of this experiment, the lack of dose effects allows us to compare SI and DI inoculations, and interpret the differences as the effects of increasing parasite genetic diversity. We believe that dose effects over this dose range were too weak to have confounded the results of our study.

Mixed versus pure inoculations

\section{Parasite fitness components}

In horizontal treatments, infectivity was higher in DI than in SI inoculations (Fig. 2a). Similar results have been reported for other endoparasites (e.g. cestodes, Davies et al. 2002; Wedekind and Ruetschi 2000; endophytes, Wille et al. 2002). This result may be caused by different mechanisms. First, in the absence of dose effects and in the presence of genetic variation for infectivity among isolates, one would expect that the overall infectivity of DI is characterized by the infectivity of the more infectious isolate. In our data, however, infectivity of DI was still higher than that of the more infectious isolate of each pair (Fig. 4a), suggesting that at least some of the increase is due to the interaction between parasite isolates at the moment of infection. Alternatively, challenging hosts with more than one parasite genotype might reduce their overall resistance (e.g. cestodes infecting copepods, Wedekind 1997). 


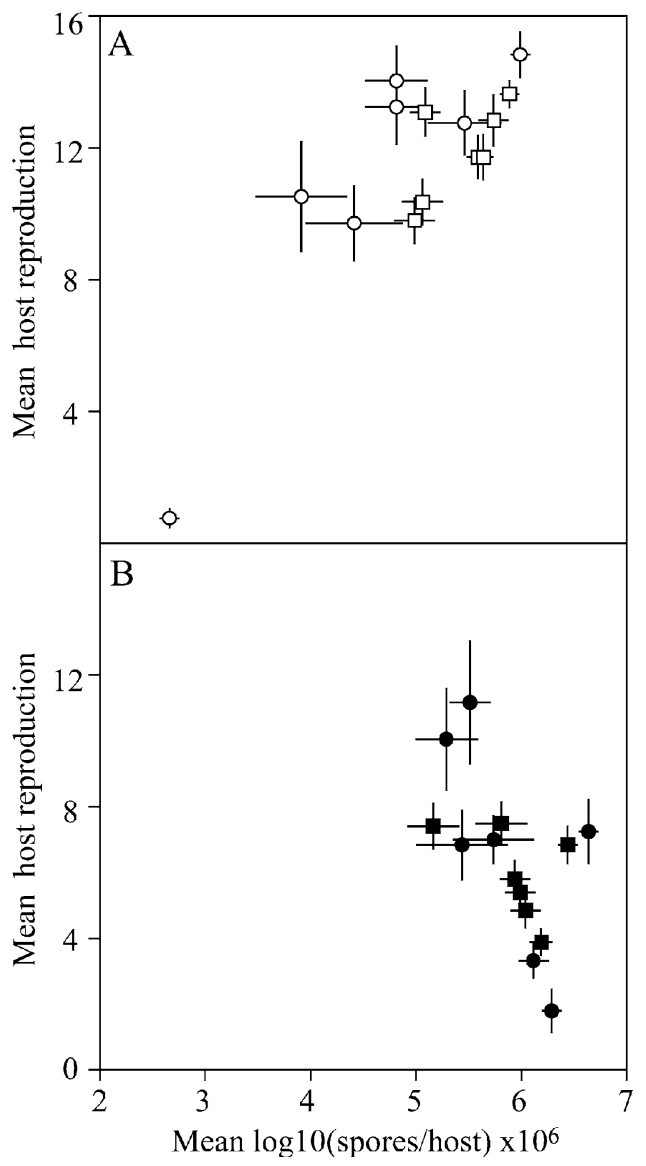

Fig. 3a,b Relationships between mean host reproduction and mean spore load across isolate combinations. Points represent the mean number of offspring produced by hosts infected with an isolate combination versus the mean spore load $\left(\log _{10}\right.$ transformed). a Horizontal infections (white symbols); b vertical infections (black symbols). The relationships are shown for SI (circles) and DI (squares) treatments. For abbreviations, see Fig. 2

Regardless of the mechanism, our results suggest that infection with a parasite strain may increase the susceptibility of the host to another parasite strain in this system. Recent theoretical work suggests that this is a necessary condition for the evolution of higher withinhost growth and/or increased host mortality rate due to the presence of more than one parasite genotype (in the case of superinfection, Day and Proulx 2004; Gandon et al. 2000).

Likewise, in horizontally infected hosts, spore load was higher in DI than in SI inoculations (Fig. 2b). As spores of different isolates were indistinguishable, we cannot conclude that DI inoculations actually resulted in double-isolate infections. It is possible that when two isolates are mixed during host inoculation, one of them will prevail later during the infection (e.g. Wille et al. 2002). In this case, the prevailing isolate may be the one with the highest within-host reproduction or competitive ability. In our data, the spore load of DI inoculations was still higher (overall) than that of the isolates with higher spore load when infected singly (Fig. 4b). The observed overall increase in spore load due to mixed inoculations, however, could still be explained in a competitive-displacement scenario if the "winner" isolate reacted plastically to the presence of genetically different parasites, or if there was selection within the isolate for genotypes of higher spore production when parasite diversity increased. Competitive displacement in mixed inoculations has been shown for endophytes (Wille et al. 2002), algae (Bell 1991), and during virus evolution (Clarke et al. 1994). For its demonstration it is required to distinguish between the genotypes. The development of genotype-specific markers in $O$. bayeri would be necessary to determine if our results are truly the result of competitive displacement.

It is unlikely that the observed higher infectivity and spore load are simply a consequence of a higher number of infective spores in DI inoculations. If the increase in infectivity observed in the DI inoculations was due to a higher number of infective spores, consequently leading to higher spore loads, we would expect to find similar results when increasing the inoculum size. To achieve a higher infectivity in double inoculations solely due to an increase of infective spores in this scenario would imply that by combining half the dose of two isolates we are increasing the proportion of infective spores in the combination, as compared to a full dose of a single isolate.

\section{Host fitness components}

Competitive displacement in parasites, where one genotype takes over due to its higher within-host reproduction, could produce an increase in the pathogenicity, either by decreasing host reproduction or longevity, as one would expect that more resources are used by the parasite (Herre 1995). Our results do not confirm this expectation, as neither a decrease in host reproduction nor in longevity was detected. Two alternative hypotheses could explain this. First, the prevailing isolate could be the most efficient in resource use, with a high spore production and a low impact on host fitness. This would lead to a positive correlation between spore production and host reproduction across isolates, in the DI treatments. Figure $3 \mathrm{a}$ shows such correlation. In natural conditions we would expect that parasite genotypes that are more efficient in their resource use will outcompete less efficient genotypes. In this experiment, however, parasite isolates from different host populations (with potentially different evolutionary histories) were placed together in a single host clone, which may explain the existence of such positive correlation. If this scenario was true, using different host genotypes should render different outcomes, as different parasite genotypes would likely do better in different hosts.

Alternatively, a combination of higher spore production and no evident changes in virulence is also in accordance with the idea that mixtures of genotypes competing for a limited resource might be more productive than pure populations of their components, as 


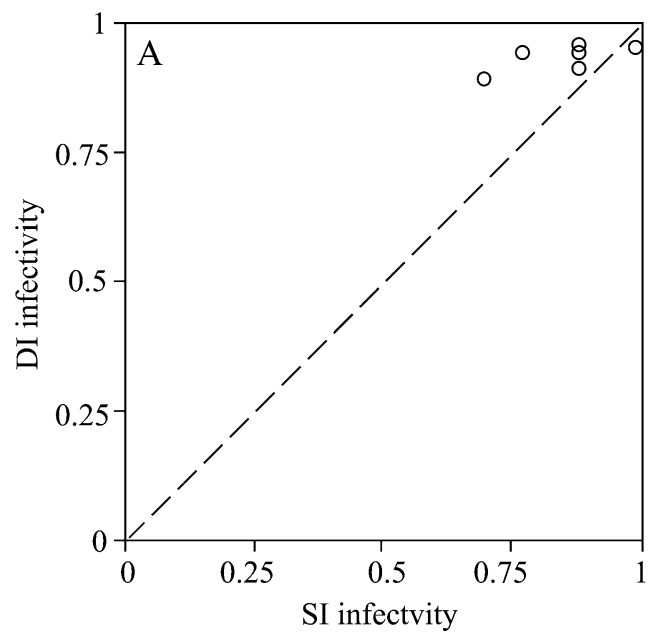

Fig. 4a,b Parasite infectivity and spore load of six $O$. bayeri isolates when infected singly or when paired with isolates with lower infectivity or spore load. Points represent the mean values for each isolate when inoculated together with another isolate of lower infectivity or spore load (DI, vertical axis) versus the mean values

Table 3 Paired $t$-tests of the differences between DI and SI means for each parasite isolate, using the SI isolate of the pair with the highest value for each variable. The percentage of difference relative to the observed values, its direction and the test results are shown for $\mathrm{h}$ and $\mathrm{v}$ treatments. $d f=5$ ( 6 pure isolates -1$)$ in all tests. Significant effects in italics. For abbreviations, see Table 2

\begin{tabular}{lllll}
\hline & & $\% \Delta$ & $|t|$-ratio & $P>t$ \\
\hline Infectivity & $\mathrm{h}$ & +8.92 & 2.31 & 0.034 \\
Total spore load & $\mathrm{h}$ & +8.93 & 1.91 & 0.057 \\
& $\mathrm{~V}$ & -2.17 & 0.78 & 0.77 \\
\hline
\end{tabular}

their resource use might be not be completely overlapping. In such a scenario, the coexistence of genetically different isolates with partially non-overlapping use of the resource will produce more spores in total than if they were all genetically similar. Parasite complementation has been shown in multiple infections with different species of microsporidia (e.g. parasites of the gypsy moth, Solter et al. 2002), where each species specialises in a subset of the resources provided by the host. This second hypothesis cannot be excluded in our experiment.

In parasites such as $O$. bayeri, that release their spores only after the host's death, an increase in mortality could be adaptive for genotypes with high within-host growth rates, as their spores would be released in the environment before those from other "slower" parasite genotypes. The within-host dynamics of this parasite show that the production of spores obeys a saturating function (Vizoso and Ebert 2004), suggesting that parasites that do not kill their hosts shortly after the rate of spore production has slowed down (around days 25-30 in our case) could be outcompeted by parasites that kill their hosts around that time (e.g. Ebert and Weisser 1997). In such a scenario, the evolution of parasite traits that directly reduce host longevity may occur (Day 2002b). The

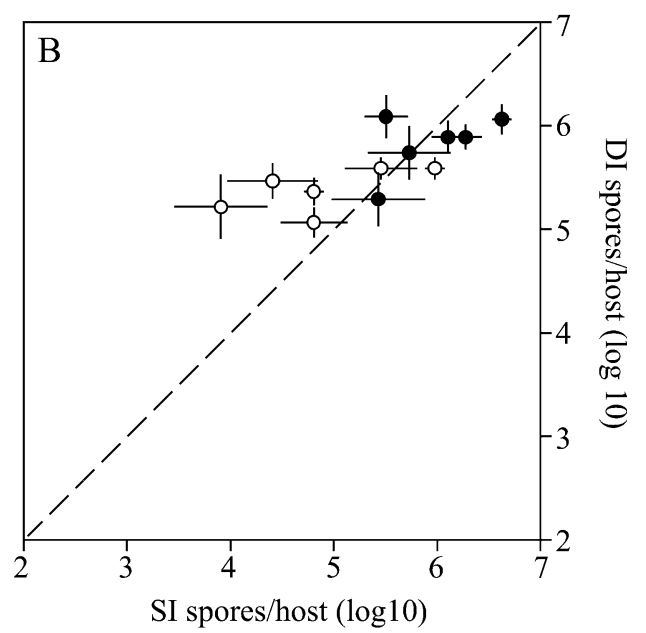

when infected singly (SI, horizontal axis). DI versus SI values shown for horizontal (open circles) and vertical (filled circles) treatments. Dashed lines indicate DI $=$ SI. a Parasite infectivity (infected/inoculated hosts); b parasite spore load (number of spores/host). For abbreviations, see Fig. 2

effect of multiple infections in such parasites has not been studied, as models usually assume a continuous release of infective propagules. The uncoupling of spore production and parasite-induced mortality in our results may suggest that there are indeed such mechanisms for host killing in this system, which are phenotypically invariant. This suggestion, however, must be taken with care, as $O$. bayeri also transmits vertically.

The increase in spore production in DI inoculations was not seen in vertical infections. This may be explained by an inherent difference between the mechanisms of horizontal and vertical infection. Vertical infection of $O$. bayeri occurs before the embryos are released into the brood chamber, and likely via a transovarial mechanism (Vizoso et al., in press). In most studied microsporidia, transovarial transmission restricts the number of parasites infecting the egg or embryo, as it occurs through a few cells surrounding the egg (Becnel and Andreadis 1999; Dunn and Smith 2001). Thus, even if the mother is infected with several parasite genotypes, only a subset of them may infect a particular egg. Which isolate has a higher transmission to the offspring will thus depend on which isolate gains more access to the ovary. It is likely that different parasite genotypes differ in their ability to parasitise the ovary. We would thus expect that the genetic diversity of vertical infections is generally lower than that of horizontal infections, which could explain the reduced difference in spore load between DI and SI inoculations when the infection occurred vertically.

Parasite plastic responses might be adaptive if the level of competition experienced in the natural environment is variable (Taylor et al. 1997). The metapopulation from which the D. magna and the $O$. bayeri used here are derived from is highly variable in many respects. Host populations are frequently invaded by other Daphnia genotypes, which may also carry parasites with 
them (Ebert et al. 2001; Haag et al. 2002). As shown by the differences among parasite isolates from different ponds, there is genetic variation in host exploitation and spore production. Thus it seems likely that parasites will encounter variable degrees of competition with other genotypes invading from other populations, and plastic responses to increases in within-host competition might be advantageous.

In summary, inoculations with higher parasite genetic diversity can increase the total parasite productivity without significantly increasing the harm to their hosts. This was not the result of changes in the parasite dose, which had no effect on the parameters measured. Vertical transmission seems to have diminished the effect of mixed inoculations, suggesting a limit to the parasite diversity in this route. The increase in parasite productivity without an associated increase in its pathogenicity could potentially reflect competitive displacement between parasite isolates, or parasite complementation. As mentioned in the Introduction, most of the theoretical work on the effect of multiple infections has focused on the evolution of parasite traits, expanding to include how changes in host life history may affect parasite fitness. In this light, and defining virulence as proportional to the increase in within-host parasite growth, our results support the expectation that multiple infections can lead to increased virulence. In many verbal arguments and some studies, however, the definition of virulence is the parasite-induced reduction in host fitness, what we called pathogenicity. Using this definition, our results suggest that multiple infections could lead to an increase in parasite reproduction without significantly increasing its negative effects on host fitness, and thus virulence. From an evolutionary point of view, we believe that different fitness components of both the parasite and the host are relevant for host-parasite coevolution. Moreover, aspects such as the dynamics of spore release and the existence of vertical transmission should also be taken into account. We hope that our results will stimulate the inclusion of host fitness components and other host and parasite life history traits in models on the evolution of virulence under multiple infections.

Acknowledgements D. B. V. thanks L. Signarski for invaluable help in the laboratory and for carrying on with the measurements during the last stage of the experiment; O. Kaltz, and L. Schärer for discussions on the analyses; J. Koella, J. Kurtz, S. Schjørring, T. Steinger, and T. Day for helpful comments on an earlier version of the manuscript. D. B. V. also thanks Weezer and NIN for timing the experiments. The laboratory material was financed by the Swiss National Funds. D. B. V. was supported by the Roche Research Foundation (Switzerland; grant 2002-68). The experiments comply with the current Swiss laws for animal manipulation.

\section{References}

Antia R, Nowak MA, Anderson RM (1996) Antigenic variation and the within-host dynamics of parasites. Proc Natl Acad Sci USA 93:985-989 van Baalen M (2000) Parent-to-offspring infection and the struggle for transmission. In: Poulin R, Moran S, Skorping A (eds) Evolutionary biology of host-parasite relationships: theory meets reality. Elsevier, Amsterdam, pp 97-116

van Baalen M, Sabelis MW (1995) The dynamics of multiple infection and the evolution of virulence. Am Nat 146:881-910

Becnel JJ, Andreadis TG (1999) Microsporidia in insects. In: Wittner M, Weiss LM (eds) The microsporidia and microsporidiosis. American Society for Microbiology, Washington, D.C., pp 447-501

Bell G (1991) The ecology and genetics of fitness in chlamydomonas. 4. The properties of mixtures of genotypes of the same species. Evolution 45:1036-1046

Bonhoeffer S, Nowak MA (1994) Mutation and the evolution of virulence. Proc R Soc Lond Ser B 258:133-140

Bremermann HJ, Pickering J (1983) A game-theoretical model of parasite virulence. J Theor Biol 100:411-426

Brown SP, Hochberg ME, Grenfell BT (2002) Does multiple infection select for raised virulence?. Trends Microbiol 10:401405

Bull JJ (1994) Perspective-virulence. Evolution 48:1423-1437

Bull JJ, Molineux IJ, Rice WR (1991) Selection of benevolence in a host-parasite system. Evolution 45:875-882

Clarke DK, Duarte EA, Elena SF, Moya A, Domingo E, Holland J (1994) The red queen reigns in the kingdom of RNA viruses. Proc Natl Acad Sci USA 91:4821-4824

Davies CM, Fairbrother E, Webster JP (2002) Mixed strain schistosome infections of snails and the evolution of parasite virulence. Parasitology 124:31-38

Day T (2002a) On the evolution of virulence and the relationship between various measures of mortality. Proc R Soc Lond Ser B 269

Day T (2002b) Virulence evolution via host exploitation and toxin production in spore-producing pathogens. Ecol Lett 5:471-476

Day T, Proulx SR (2004) A general theory for the evolutionary dynamics of virulence. Am Nat 163:E40-E63

Dunn AM, Smith JE (2001) Microsporidian life cycles and diversity: the relationship between virulence and transmission. Microbes Infect 3:381-388

Ebert D (1994) Virulence and local adaptation of a horizontally transmitted parasite. Science 265:1084-1086

Ebert D, Weisser WW (1997) Optimal killing for obligate killers: the evolution of life histories and virulence of semelparous parasites. Proc R Soc Lond Ser B 264:985-991

Ebert D, Zschokke-Rohringer CD, Carius HJ (1998) Within- and between-population variation for resistance of Daphnia magna to the bacterial endoparasite Pasteuria ramosa. Proc R Soc Lond Ser B 265:2127-2134

Ebert D, Zschokke-Rohringer C, Carius HJ (2000) Dose effects and density-dependent regulation of two microparasites of Daphnia magna. Oecologia 122:200-209

Ebert D, Hottinger JW, Pajunen VI (2001) Temporal and spatial dynamics of parasite richness in a Daphnia metapopulation. Ecology 82:3417-3434

Ebert D, Haag C, Kirkpatrick M, Riek M, Hottinger JW, Pajunen VI (2002) A selective advantage to immigrant genes in a Daphnia metapopulation. Science 295:485-488

Frank SA (1992) A kin selection model for the evolution of virulence. Proc R Soc Lond Ser B 250:195-197

Frank SA (1996) Models of parasite virulence. Q Rev Biol 71:37-78

Gandon S, Jansen VAA, van Baalen M (2000) Host life-history and the evolution of virulence. Evolution 55:1056-1062

Green J (1957) Parasites and epibionts of Cladocera in rock pools of Tvärminne archipelago. Arch Soc Zool Bot Fenn 12:5-12

Haag CR, Hottinger JW, Riek M, Ebert D (2002) Strong inbreeding depression in a Daphnia metapopulation. Evolution 56:518-526

Hellriegel B (1992) Modelling the immune-response to malaria with ecological concepts - short-term behavior against long-term equilibrium. Proc R Soc Lond Ser B 250:249-256

Herre EA (1993) Population-structure and the evolution of virulence in nematode parasites of fig wasps. Science 259:1442-1445 
Herre EA (1995) Factors affecting the evolution of virulence: nematode parasites of fig wasps as a case study. Parasitology 111:S179-S191

Klüttgen B, Dülmer U, Engels M, Ratte HT (1994) ADaM, an artificial freshwater for the culture of zooplankton. Water Res 28:743-746

Knolle H (1989) Host density and the evolution of parasite virulence. J Theor Biol 136:199-207

May RM, Nowak MA (1995) Coinfection and the evolution of parasite virulence. Proc R Soc Lond Ser B 261:209-215

Perlman SJ, Jaenike J (2003) Evolution of multiple components of virulence in Drosophila- nematode associations. Evolution $57: 1543-1551$

Poulin R, Combes C (1999) The concept of virulence: Interpretations and implications. Parasitol Today 15:474-475

Read AF (1994) The evolution of virulence. Trends Microbiol 2:73-76

Read AF, Taylor LH (2001) The ecology of genetically diverse infections. Science 292:1099-1102

SAS Institute (2000) JMP, 4th edn. Cary, N.C.

Schjørring S, Koella JC (2003) Sub-lethal effects of pathogens can lead to the evolution of lower virulence in multiple infections. Proc R Soc Lond Ser B 270:189-193

Solter LF, Siegel JP, Pilarska DK, Higgs MC (2002) The impact of mixed infection of three species of microsporidia isolated from the gypsy moth, Lymantria dispar L. (Lepidoptera : Lymantriidae). J Invert Pathol 81:103-113

Taylor LH, Walliker D, Read AF (1997) Mixed-genotype infections of malaria parasites: within-host dynamics and transmission success of competing clones. Proc R Soc Lond Ser B 264:927-935
Turner PE, Chao L (1999) Prisoner's dilemma in an RNA virus. Nature 398:441-443

Vizoso DB (2003) Phenotypic plasticity in a host-parasite interaction with horizontal and vertical transmission: a study on infection routes and genetic diversity of Octosporea bayeri (Microsporidia) in Daphnia magna (Cladocera). Institut für Biologie, Ökologie und Evolution. Universität Freiburg, Freiburg

Vizoso DB, Ebert D (2004) Within-host dynamics of a microsporidium with horizontal and vertical transmission: Octosporea bayeri in Daphnia magna. Parasitology 128:31-38

Vizoso DB, Ebert D (in review) Phenotypic plasticity of host-parasite interactions in response to the route of infection

Vizoso DB, Lass S, Ebert D (in press) Different mechanisms of transmission of the microsporidium Octosporea bayeri: a cocktail of solutions for the problem of parasite permanence. Parasitology

Wedekind C (1997) The infectivity, growth, and virulence of the cestode Schistocephalus solidus in its first intermediate host, the copepod Macrocyclops albidus. Parasitology 115:317-324

Wedekind C, Ruetschi A (2000) Parasite heterogeneity affects infection success and the occurrence of within-host competition: an experimental study with a cestode. Evol Ecol Res 2:10311043

Wille P, Boller T, Kaltz O (2002) Mixed inoculation alters infection success of strains of the endophyte Epichloe bromicola on its grass host Bromus erectus. Proc R Soc Lond Ser B 269:397-402 